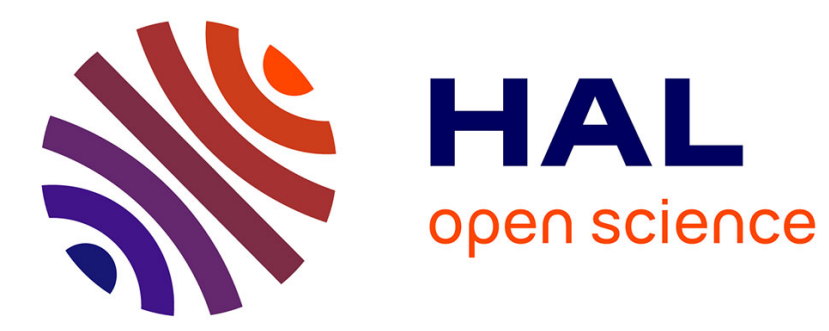

\title{
Hydrodynamic response of a trapped superfluid to a periodic perturbation
}

Shuwei Jin, Sébastien Laurent, Frédéric Chevy

\section{To cite this version:}

Shuwei Jin, Sébastien Laurent, Frédéric Chevy. Hydrodynamic response of a trapped superfluid to a periodic perturbation. 2019. hal-01713122

\section{HAL Id: hal-01713122 \\ https://hal.science/hal-01713122}

Preprint submitted on 21 Oct 2019

HAL is a multi-disciplinary open access archive for the deposit and dissemination of scientific research documents, whether they are published or not. The documents may come from teaching and research institutions in France or abroad, or from public or private research centers.
L'archive ouverte pluridisciplinaire HAL, est destinée au dépôt et à la diffusion de documents scientifiques de niveau recherche, publiés ou non, émanant des établissements d'enseignement et de recherche français ou étrangers, des laboratoires publics ou privés. 


\title{
Hydrodynamic response of a trapped superfluid to a periodic perturbation
}

\author{
Shuwei Jin ${ }^{1}$, a, Sébastien Laurent ${ }^{1}$, and Frédéric Chevy ${ }^{1}$ \\ Laboratoire Kastler Brossel, ENS-PSL Research University, CNRS, Sorbonne Université, \\ Collège de France, 24 rue Lhomond, 75005 Paris, France
}

\begin{abstract}
In this article we discuss the hydrodynamic response of a superfluid to a periodic drive. Using a WKB description of the hydrodynamic modes of the system, we show that contrary to Landau's argument, there is no critical velocity, but that the dissipated power nevertheless increases rapidly when the maximum velocity of the perturbation reaches the central sound velocity of the superfluid.
\end{abstract}

\section{Introduction}

The existence of frictionless flow in quantum fluids at low temperature is one of the most dramatic macroscopic manifestations of quantum mechanics. As originally conjectured by Landau [1], one key feature of superfluid flows is the existence of a critical velocity above which dissipation nevertheless arises and in its simplest form, this critical velocity marks the threshold beyond which a moving impurity can generate a stationary wake of elementary excitations (phonons or rotons for helium). As such this physical phenomenon is similar to the Cherenkov radiation in electrodynamics [2] or to the generation of surface waves by an object moving at the surface of liquid [3, 4]. Experimental evidences for the existence of this critical velocity were presented in superfluid helium[5], ultracold gases [6-11] and polariton condensates [12]. However it was also rapidly pointed out that in actual experiments, additional decay channels were lowering the predicted critical velocity. It was first observed that in the case of a strong perturbation, vortex shedding could supersede generation of elementary exitations [13-17], while in attractive Fermi gases paired-breaking excitations can become dominant in the weakly interacting limit [18]. In trapped gases, density inhomogeneities $[19,20]$ and thermal fluctuations [21-23] can also strongly decrease the critical velocity. Finally, the threshold is even completely smeared out when the impurity does not move at a constant velocity $[24,22]$, just like accelerated charged particles radiate electromagnetic waves at any speed [25].

Previous studies were focusing on mean-field descriptions of the system and in this paper we investigate theoretically the hydrodynamic response of an arbitrary superfluid to a time-periodic external potential. We restrict our study to the case of an elongated superfluid in which the transverse degrees of freedom can be integrated out. Using the WKB approximation we determine the high-energy modes of the superfluid for an arbitrary equation of state. In particular we show that the high-frequency

\footnotetext{
${ }^{a}$ e-mail: shuwei.jin@lkb.ens.fr
} 
spectrum has a phonon-like dispersion relation. Using those results we compute the power transferred from a stirring potential to the superfluid and we confirm the absence of critical velocity for an accelerated impurity.

\section{Hydrodynamic modes of a superfluid in an elongated potential}

\subsection{Linearized equations}

In this first paragraph, we recall some general results on the hydrodynamic modes of a superfluid. We consider a superfluid trapped in an elongated harmonic potential. Integrating-out the transverse degrees of freedom [26], we describe its dynamics by a semi-classical one-dimensional Hamiltonian

$$
H=\int d z\left(\frac{\hbar^{2}}{2 m}\left(\partial_{z} \chi\right)^{2}+e(n)+n V(z)\right),
$$

where $z$ is the weak-trapping direction, $m$ the mass of the particles, $n$ the $1 \mathrm{D}$ particledensity, $\chi$ the phase of the order parameter, $e(n)$ the $1 \mathrm{D}$ energy-density, and $V(z)=$ $m \omega_{z}^{2} z^{2} / 2$ the trapping potential along $z$. Writing Hamilton's equations for the conjugate variables $n$ and $\chi$ yields the usual hydrodynamic equations

$$
\begin{aligned}
\partial_{t} n+\partial_{z}(n v) & =0, \\
\hbar \partial_{t} \chi+m \frac{v^{2}}{2} & =-V(z)-\mu(n),
\end{aligned}
$$

where $\mu(n)=\partial_{n} e(n)$ is the chemical potential of the system and $v=\hbar \partial_{z} \chi / m$ the superfluid velocity. In stationary regime, the density is constant and the phase $\chi$ varies linearly with time as $\chi_{\mathrm{s}}=-\mu_{0} t / \hbar$. Inserting these expressions in Eq. $(2,3)$ yields the Local Density Approximation (LDA) equation for the density $\mu\left(n_{\mathrm{s}}\right)+V(z)=\mu_{0}$. In particular, assuming that $\mu(n=0)=0$, the boundaries of the cloud are located in $z= \pm Z$, with $m \omega_{z}^{2} Z^{2} / 2=\mu_{0}$.

We now focus on the solutions of Eq. $(2,3)$ close to equilibrium. We take $\chi=\chi_{\mathrm{s}}+\varphi$ and $n=n_{\mathrm{s}}+\delta n$ with $\varphi \ll \chi_{\mathrm{s}}$ and $\delta n \ll n_{\mathrm{s}}$. Expanding the hydrodynamic equations to first order in perturbation, Eq. $(2,3)$ can be recast as

$$
\begin{aligned}
\partial_{t} \delta n & =-\partial_{z}\left(n_{\mathrm{s}} v\right), \\
\hbar \partial_{t} \varphi & =-\frac{\partial \mu_{\mathrm{s}}}{\partial n_{\mathrm{s}}} \delta n,
\end{aligned}
$$

where $\mu_{\mathrm{s}}$ stands for $\mu\left(n_{\mathrm{s}}\right)$. Taking $\delta \mu=\partial_{n_{\mathrm{s}}} \mu_{\mathrm{s}} \delta n$, we finally obtain a wave-like equation

$$
\partial_{t}^{2} \delta \mu-\frac{1}{m}\left(\frac{\partial \mu_{\mathrm{s}}}{\partial n_{\mathrm{s}}}\right) \partial_{z}\left(n_{\mathrm{s}} \partial_{z} \delta \mu\right)=0
$$

The eigenmodes of the system correspond to time-oscillating solutions $\delta \mu(z, t)=$ $\psi_{q}(z) e^{-i \omega_{q} t}$, where $\psi_{q}$ and $\omega_{q}$ are solutions of the Eigenvalue problem $[27,26]$

$$
\omega_{q}^{2} \psi_{q}=\mathcal{L}\left[\psi_{q}\right]
$$

where $q$ is an integer labelling the eigenmodes and $\mathcal{L}$ the linear operator defined by

$$
\mathcal{L}[\psi]=-\frac{1}{m}\left(\frac{\partial \mu_{\mathrm{s}}}{\partial n_{\mathrm{s}}}\right) \partial_{z}\left(n_{\mathrm{s}} \partial_{z} \psi\right) .
$$


$\mathcal{L}$ is hermitian for the scalar product

$$
(f \mid g)=\int d z \frac{\partial n_{\mathrm{s}}}{\partial \mu_{\mathrm{s}}} f(z)^{*} g(z),
$$

and, since by thermodynamic stability $\partial_{n_{\mathrm{s}}} \mu_{\mathrm{s}}>0$, it is also a positive operator, meaning that the hydrodynamic eigenfrequencies $\omega_{q}$ are all real.

In the literature Eq. (7) was solved in the case of a polytropic equation of state $\mu=\alpha n^{\gamma}$, where $\alpha$ and $\gamma$ are two constant parameters (in the case of a weakly interacting Bose-Einstein condensate, $\gamma=1 / 2$, while for a unitary Fermi gas [28, 29], $\gamma=2 / 5$. For a 3D polytropic gas characterzed by a 3D polytropic exponent $\gamma_{3 D}$ and confined in a harmonic potential, we have $1 / \gamma=1 / \gamma_{3 D}+1$, hence $\gamma<1$ ). In this case, the eigenfunctions are polynomials ${ }^{1}$ and the corresponding eigenvalues are given by

$$
\omega_{q}=\omega_{z} \sqrt{q[2+\gamma(q-1)] / 2} .
$$

At large frequencies, we recover an acoustic-like linear spectrum since two subsequent modes are separated by a constant frequency gap $\Delta \omega=\omega_{z} \sqrt{\gamma / 2}$. Moreover, take $c_{1 D}$ the sound velocity in absence of axial trapping, we have [28]

$$
c_{1 D}=\sqrt{\frac{n}{m} \frac{\partial \mu}{\partial n}}=\sqrt{\frac{\gamma \mu}{m}} .
$$

We can then recast the high-frequency dispersion relation in a Fabry-Perot-like form

$$
\omega_{q} \simeq 2 \pi\left(q+q_{0}\right) \frac{c_{1 D}^{(0)}}{L_{\mathrm{eff}}}
$$

where $c_{1 \mathrm{D}}^{(0)}$ is the local $1 \mathrm{D}$-sound-velocity at the trap center, $L_{\mathrm{eff}}=2 \pi Z$ is the effective acoustic length of the cloud and $q_{0}=1 / \gamma-1 / 2$ is a "quantum defect".

\subsection{WKB Approximation}

We now generalize the previous results to an arbitrary equation of state $\mu(n)$ and we show that the high-energy hydrodynamic spectrum still follows a phonon-like dispersion relation similar to (12). Take $\psi_{q}=A(z) e^{i \theta(z)}$ where $A$ and $\theta$ are two real functions. Eq. (7) turns then into the following set of equations:

$$
\begin{aligned}
m \omega_{q}^{2} A & =-\left(\partial_{z} \mu_{\mathrm{s}}\right)\left(\partial_{z} A\right)-n_{\mathrm{s}}\left(\partial_{z}^{2} A\right)\left(\partial_{n_{\mathrm{s}}} \mu_{\mathrm{s}}\right)+n_{\mathrm{s}} A\left(\partial_{z} \theta\right)^{2}\left(\partial_{n_{\mathrm{s}}} \mu_{\mathrm{s}}\right), \\
0 & =A\left(\partial_{z} n_{\mathrm{s}}\right)\left(\partial_{z} \theta\right)+2 n_{\mathrm{s}}\left(\partial_{z} \theta\right)\left(\partial_{z} A\right)+A n_{\mathrm{s}}\left(\partial_{z}^{2} A\right) .
\end{aligned}
$$

Eq. (14) is readily integrated and yields the conservation of the probability current $n_{\mathrm{s}} A^{2} k(z)=$ const, with $k(z)=\partial_{z} \theta$ the local wave-vector. For a homogeneous system, the solutions of the wave equation are plane waves characterized by a uniform wavevector $k$. In a trap, the spatial dependence of the local density implies that $k(z)$ varies spatially over a typical length scale given by the size $Z$ of the cloud. In the WKB approximation, we consider high-frequency modes and we assume that the typical

\footnotetext{
1 More precisely Gegenbauer polynomials, which are defined as the family of orthogonal polynomials for the weight function $w(z)=\left(Z^{2}-z^{2}\right)^{\alpha}$ - in the present case $\alpha=1 / \gamma-1$ [30].
} 


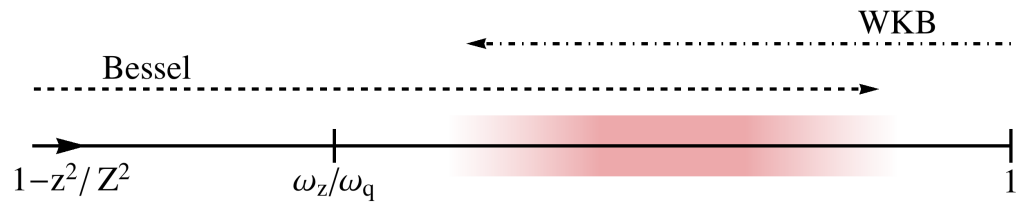

Fig. 1. Domain of validity of the two asymptotic regimes. The red shaded area shows the region where they are both valid and where the quantization of the frequencies can be made by matching the two asymptotic expressions.

wavelength $k^{-1}$ of the excitation is much smaller than $Z$. In this limit we can neglect higher-order spatial-derivatives in Eq. (13) which simplifies into

$$
\omega_{q}^{2}=\frac{n_{\mathrm{s}}}{m} \frac{\partial \mu_{\mathrm{s}}}{\partial n_{s}} k(z)^{2}
$$

hence

$$
\theta(z)=\theta(0) \pm \int_{0}^{z} d z^{\prime} \frac{\omega_{q}}{c_{1 \mathrm{D}}(z)}
$$

with $m c_{1 \mathrm{D}}(z)^{2}=n_{\mathrm{s}} \partial_{n_{\mathrm{s}}} \mu_{\mathrm{s}}$ the local sound-velocity and the \pm sign corresponds to the direction of propagation of the wave. The general solution is the sum of left and right-propagating waves and we can finally write that

$$
\psi_{q} \propto \frac{1}{\sqrt{n_{\mathrm{s}}(z) k(z)}} \cos \left(\int_{0}^{z} \frac{\omega d z^{\prime}}{c_{1 \mathrm{D}}\left(z^{\prime}\right)}+\theta(0)\right) .
$$

(Note that in practice, $\theta(0) \equiv 0[\pi / 2]$ since the solution must be either odd or even in a harmonic potential).

The frequency quantization arises from the boundary conditions at the cloud edge. In this region, the density and the sound velocity vanish and we can no longer approximate $\psi_{q}$ by a local plane wave. We obtain another asymptotic expansion valid for $z \rightarrow Z$, by expanding Eq. (7) as

$$
\omega_{q}^{2} \psi_{q}=\frac{2 \mu_{0}}{m Z} \partial_{z} \psi_{q}-\left(\frac{2 \gamma \mu_{0}}{m}\right)\left(1-\frac{z}{Z}\right) \partial_{z}^{2} \psi_{q}
$$

where we have used the LDA condition $\mu_{z}=\mu_{0}\left(1-z^{2} / Z^{2}\right) \simeq 2 \mu_{0}(1-z / Z)$ and we have assumed that for small densities we could approximate the equation of state of the superfluid by a polytropic expression $\mu_{\mathrm{s}} \simeq \alpha n_{\mathrm{s}}^{\gamma}$.

This equation can be solved analytically in terms of Bessel functions of the first kind $J_{\alpha}$, and the regular solution is given by

$$
\psi_{q} \propto \frac{J_{1 / \gamma-1}\left(\omega Z \sqrt{\frac{2 m}{\mu_{0}}(1-z / Z)}\right)}{\sqrt{1-z / Z^{1 / \gamma-1}}} .
$$

Expanding this solution far from the boundary yields the asymptotic expression [30]

$$
\psi_{q} \propto \frac{\cos \left(\omega_{q} Z \sqrt{\frac{2 m}{\mu_{0}}(1-z / Z)}-\frac{\pi}{2 \gamma}+\frac{\pi}{4}\right)}{\sqrt{1-z / Z^{1 / \gamma-1 / 2}}} .
$$

The quantization condition is obtained by matching the two asymptotic expressions (17) and (20). Expanding the phase of Eq. (17) close to $z=Z$, we indeed see that 


$$
\int_{0}^{z} \frac{\omega d z^{\prime}}{c_{1 \mathrm{D}}\left(z^{\prime}\right)}=\int_{0}^{Z} \frac{\omega d z^{\prime}}{c_{1 \mathrm{D}}\left(z^{\prime}\right)}+\int_{Z}^{z} \frac{d z^{\prime}}{c_{1 \mathrm{D}}\left(z^{\prime}\right)} \simeq \int_{0}^{Z} \frac{\omega d z^{\prime}}{c_{1 \mathrm{D}}\left(z^{\prime}\right)}-\omega Z \sqrt{\frac{2 m}{\mu_{0}}(1-z / Z)},
$$

where we have used the polytropic expression of the sound velocity at vanishingly small density.

We can match this expression to the phase appearing in Eq. (20) in the range $1 \gg(1-z / Z) \gg \omega_{z}^{2} / \omega_{q}^{2}$ (See Fig. 1). Indeed, on the one hand Eq. (20) is obtained under the assumption that i) we are close to the edge $((1-z / Z) \ll 1)$, and ii) the argument appearing in Bessel function is large, hence $\omega_{q} \sqrt{(1-z / Z) 2 m / \mu_{0}} \gg 1$. Since $\mu_{0}=m \omega_{z}^{2} Z^{2} / 2$, it means that $(1-z / Z) \gg \omega_{z}^{2} / \omega_{q}^{2}$. On the other hand, the WKB expression was derived by neglecting the terms $\left(\partial_{z} \mu_{\mathrm{s}}\right)\left(\partial_{z} A\right)$ and $n_{\mathrm{s}}\left(\partial_{z}^{2} A\right) \partial_{n_{s}} \mu_{\mathrm{s}}$ with respect to $m \omega_{q}^{2} A$ in Eq. (13). Since $A \propto 1 / \sqrt{n_{\mathrm{s}}(z) k(z)}$, it scales as a power-law with $1-z^{2} / Z^{2}$, and close to the edge, we have therefore $\left(\partial_{z} \mu_{\mathrm{s}}\right)\left(\partial_{z} A\right) \sim n_{\mathrm{s}}\left(\partial_{z}^{2} A\right) \partial_{n_{s}} \mu_{\mathrm{s}} \sim$ $m \omega_{z}^{2} A /(1-z / Z)$ hence the validity criterion $(1-z / Z) \gg \omega_{z}^{2} / \omega_{q}^{2}$. Since the expansion (21) is obtained for $(1-z / Z) \ll 1$, we deduce that Bessel and WKB expressions must match in the region $\omega_{z}^{2} / \omega_{q}^{2} \ll(1-z / Z) \ll 1$. In particular it requires the condition $\omega_{z}^{2} \ll \omega_{q}^{2}$ which is satisfied by higher-frequency modes.

Comparing Eq. (20) and (21), we see that the WKB waves are reflected at the edge of the cloud with a dephasing $\pi / \gamma-\pi / 2$. Requiring the existence of a standing wave when the excitation travels back and forth between $z= \pm Z$ finally yields the resonance condition

$$
2 \omega_{q} \int_{-Z}^{Z} \frac{d z^{\prime}}{c_{1 \mathrm{D}}\left(z^{\prime}\right)}-\frac{2 \pi}{\gamma}+\pi=2 q \pi
$$

hence the high-frequency spectrum

$$
\omega_{q}=\pi \frac{q+1 / \gamma-1 / 2}{\int_{-Z}^{Z} \frac{d z^{\prime}}{c_{1 \mathrm{D}}\left(z^{\prime}\right)}} .
$$

We recover the asymptotic expression (12) obtained for a purely polytropic gas, with the same expression for the quantum defect and a generalized effective length

$$
L_{\mathrm{eff}}=2 c_{1 D}^{(0)} \int_{-Z}^{Z} \frac{d z^{\prime}}{c_{1 \mathrm{D}}\left(z^{\prime}\right)} .
$$

Finally, if we normalize $\psi_{q}$ according to the scalar product (9), we get

$$
\psi_{q}(z)=\sqrt{\frac{2 m \Delta \omega c_{1 \mathrm{D}}(z)}{n(z)}} \cos \left[\int_{0}^{z} d z^{\prime} \frac{\omega_{q}}{c_{1 \mathrm{D}}\left(z^{\prime}\right)}+q \frac{\pi}{2}\right]
$$

where $\Delta \omega^{-1}=L_{\text {eff }} / 2 \pi c_{1 \mathrm{D}}^{(0)}$ is the density of state.

In Fig. 2 we compare the WKB wave-functions and the exact Gegenbauer polynomials in the case $\gamma=1 / 2$ (mean-field BEC) and we observe a very good agreement, even for low-order modes.

We see that for a one-dimensional trap system, the high-frequency spectrum has a phonon-like structure, and the eigenfunction can be locally described by planewaves. Note that this result is valid as long as $k R_{\perp} \ll 1$, where $R_{\perp}$ is the transverse size of the cloud. If we now take into account the transverse degrees of freedom, the dispersion relation becomes sub-linear $[27,26,20]$. 

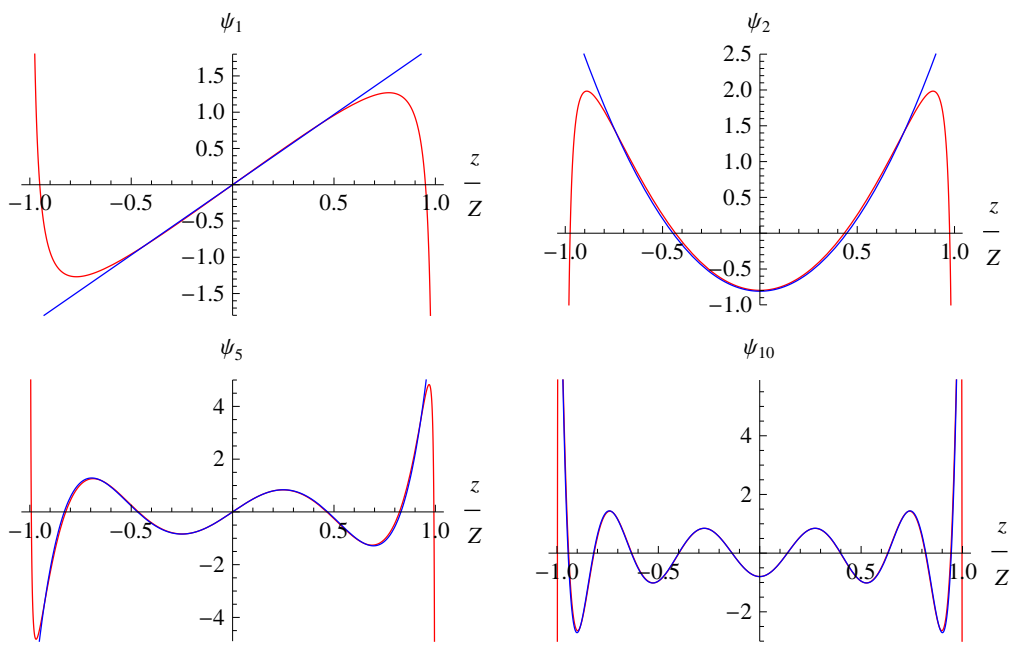

Fig. 2. Comparison between Gegenbauer polynomials (blue curves) and WKB approximation (red curves) for $\gamma=1 / 2$.

\section{Response to an external drive}

\subsection{General formalism}

We now study the excitation of the hydrodynamic modes by an external potential created by a moving laser beam oscillating at a frequency $\Omega$ along the axis of the cloud. The response of the cloud is obtained by solving the hydrodynamic equations $(2,3)$ where we add to the trapping potential an excitation term $u(z, t)=f(z-b(t)) e^{\varepsilon t}$ and $b(t)=b_{0} \cos (\Omega t)$ that we turn on adiabatically over a time $1 / \varepsilon \rightarrow \infty$ to get rid of transient evolution. The chemical potential now obeys the following equation:

$$
\partial_{t}^{2} \delta \mu+\mathcal{L}[\delta \mu]=-\mathcal{L}[u] .
$$

To solve this equation, we first note that the external drive is periodic and that both $u$ and $\delta \mu$ can be Fourier expanded as

$$
\begin{aligned}
u(z, t) & =\sum_{p, q} u_{p, q} \psi_{q}(z) e^{i(p \Omega+\varepsilon) t}, \\
\delta \mu(z, t) & =\sum_{p, q} \delta \mu_{p, q} \psi_{q}(z) e^{i(p \Omega+\varepsilon) t} .
\end{aligned}
$$

Inserting these expression in Eq. (26) we get

$$
\delta \mu_{p, q}=\frac{\omega_{q}^{2} u_{p, q}}{p^{2} \Omega^{2}-\omega_{q}^{2}-i \varepsilon \operatorname{sg}(p \Omega)} .
$$

where sg is the sign function and we have used the fact that the $\psi_{q}$ are the eigenvectors of $\mathcal{L}$ for the eigenvalue $\omega_{q}^{2}$.

The mechanical power transferred from the stirring potential to the cloud is given by the expression

$$
P=-\left\langle\int d z^{\prime} n_{\mathrm{s}}\left(z^{\prime}\right)\left(\partial_{z} u\left(z^{\prime}, t\right)\right) v\left(z^{\prime}, t\right)\right\rangle
$$


where $\langle$.$\rangle denotes the time average. Using the fact that v=\hbar \partial_{z} \varphi / m$ we get after integrating by part

$$
P=\frac{\hbar}{m}\left\langle\int d z^{\prime} \partial_{z}\left(n_{\mathrm{s}}\left(z^{\prime}\right)\left(\partial_{z} \varphi\left(z^{\prime}, t\right)\right)\right) u\left(z^{\prime}, t\right)\right\rangle=-\hbar\langle(\mathcal{L}[\varphi] \mid u)\rangle .
$$

From particle number conservation (Eq. 3), we see that $\hbar \mathcal{L}[\varphi]=\partial_{t} \delta \mu$, hence the final expression

$$
P=\left\langle\left(\partial_{t} \delta \mu \mid u\right)\right\rangle=\sum_{p, q} \frac{i p \Omega \omega_{q}^{2}\left|u_{p, q}\right|^{2}}{\omega_{q}^{2}-p^{2} \Omega^{2}+i \varepsilon \operatorname{sg}(p \Omega)} .
$$

where we have used Eq. (29) and the fact that the $\psi_{q}$ form an ortho-normalized basis for $(\mid)$. Finally, since the power is a real quantity the sum simplifies into

$$
P=\left\langle\left(\partial_{t} \delta \mu \mid u\right)\right\rangle=\pi \sum_{p, q}|p \Omega|^{3}\left|u_{p, q}\right|^{2} \delta\left(p^{2} \Omega^{2}-\omega_{q}^{2}\right) .
$$

\subsection{High-frequency drive}

We now specialize the previous expression to the case where the excitation frequency $\Omega$ is much larger than $\omega_{z}$ (this condition is indeed satisfied in [6]). This assumption leads to two simplifications: firstly, we can treat the sum over $q$ as a continuous integral, with respect to the sum over $p$. In other words, the acoustic spectrum can be considered as continuous with respect to the discrete harmonics of the excitation. Second, since $c_{1 \mathrm{D}}^{(0)} \propto \sqrt{\mu_{0} / m}$ and $\mu_{0}=m \omega_{z}^{2} Z^{2} / 2$, we see Landau's critical velocity is reached for a modulation amplitude $b_{0}=c_{1 \mathrm{D}}^{(0)} / \Omega \ll Z$, which means that the stirring potential remains localized close to the cloud center.

Before calculating explicitly the dissipated power, we first show that the Fourier components $u_{p, q}$ vanish when $p$ and $q$ have opposite parities. Indeed, we have

$$
u_{p, q}=\frac{1}{T} \int_{0}^{T} d t \int_{-Z}^{Z} d z \frac{\partial n_{\mathrm{s}}}{\partial \mu_{\mathrm{s}}} \psi_{q}(z)^{*} u\left(z-b_{0} \cos (\Omega t)\right) e^{-i p \Omega t} .
$$

where $T=2 \pi / \Omega$ is the period of the excitation. Using the symmetries $z \rightarrow-z$ and $\Omega t \rightarrow \pi-\Omega t$, we see that for an even pertubating potential $u_{p, q}(z)=(-1)^{p+q} u_{p, q}(z)$.

Since the perturbation remains localized close to the cloud center, we can use WKB approximation and replace $\psi_{q}$ by a sinusoid. For both parities of $p$, we then obtain

$$
\left|u_{p, q}\right|^{2}=\frac{2 n_{0} \Delta \omega}{m c_{1 \mathrm{D}}^{(0)}} \tilde{u}\left(\frac{\omega_{q}}{c_{1 \mathrm{D}}^{(0)}}\right)^{2} J_{p}\left(\frac{\omega_{q} b_{0}}{c_{1 \mathrm{D}}^{(0)}}\right)^{2},
$$

with $\tilde{u}(k)=\int d z \cos (k z) u(z)$. Hence the dissipated power is

$$
P=2 \pi \frac{\Omega^{2} n_{0}}{m c_{1 \mathrm{D}}^{(0)^{3}}} \sum_{p \geq 0} p^{2} \tilde{u}\left(\frac{p \Omega}{c_{1 \mathrm{D}}^{(0)}}\right)^{2} J_{p}\left(\frac{p \Omega b_{0}}{c_{1 \mathrm{D}}^{(0)}}\right)^{2} .
$$

From this expression, we deduce the absence of critical velocity for the onset of dissipation. Indeed, at low $\Omega / c_{1 \mathrm{D}}$, we can expand the previous expression as

$$
P \simeq \pi \frac{\Omega^{4} n_{0} b_{0}^{2}}{m c_{1 \mathrm{D}}^{(0)^{5}}} \tilde{u}(0)^{2} .
$$




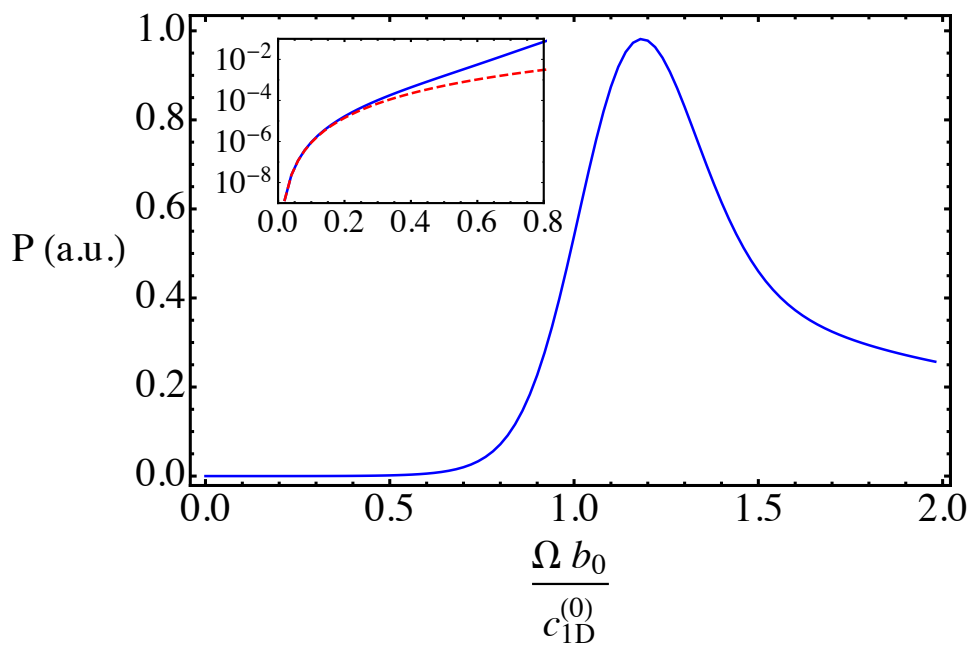

Fig. 3. Dissipated power for a gaussian perturbation of size $w=0.09 Z$ with an amplitude $b_{0}=0.4 Z$. Inset: The dissipated power is represented in log-scale to show its increase $\propto \Omega^{4}$ (red dashed curve) at low frequency.

Note that we recover here $\Omega^{4} b_{0}^{2}$ scaling characterizing the power radiated by an electrodynamic dipole [25]. We recover a trace of Landau's critical velocity by considering the limiting case of a inifinitely narrow excitation potential. In this case, we can assume that $\tilde{u}$ is constant and the dissipated power then becomes

$$
P=2 \pi \frac{\Omega^{2} n_{0}}{m c_{1 \mathrm{D}}^{(0)^{3}}} \sum_{p \geq 0} p^{2} J_{p}\left(\frac{p \Omega b_{0}}{c_{1 \mathrm{D}}^{(0)}}\right)^{2} \tilde{u}(0)^{2} .
$$

The asymptotic behaviour of Bessel functions for large orders is given by [30]

$$
\begin{aligned}
J_{\nu}(\nu \operatorname{sech}(\alpha)) & \sim \frac{e^{-\nu(\alpha-\tanh (\alpha))}}{\sqrt{2 \pi \nu \tanh (\alpha)}} \\
J_{\nu}(\nu \sec (\beta)) & \sim \sqrt{\frac{2}{\pi \nu \tan (\beta)}} \cos (\nu \tan (\beta)-\nu \beta-\pi / 4),
\end{aligned}
$$

with $\nu \rightarrow \infty$. For a maximum stirring velocity $V=\Omega b_{0}$ smaller than the sound velocity $c_{1 \mathrm{D}}^{(0)}$, we take $\operatorname{sech}(\alpha)=V / c_{1 \mathrm{D}}^{(0)}$ and we can see using Eq. (39) that the large order terms of the series decay exponentially for $p \rightarrow \infty$. By contrast, for $V>c_{1 \mathrm{D}}^{(0)}$, we take $\sec (\beta)=V / c_{1 \mathrm{D}}^{(0)}$ and using Eq. (40) we see that the terms of the series diverge as $p$ for large $p$, hence an infinite dissipated power when Landau's critical velocity is reached.

For realistic systems, this divergence is cured by the finite extension of the exciting potential, which leads to a finite dissipation for velocities larger than the sound velocity at the center of the trap, and even a decrease of the dissipation at large velocities. In Fig. 3, we illustrate this regularization by assuming that $u$ is a finite-size gaussian perturbation. We observe that due to the destructive interferences between the waves emitted by an extended object, the amount of energy dissipation occurring at $v \simeq c_{1 \mathrm{D}}^{(0)}$ decreases when the size of the object increases. For similar stirring parameters as in 
[6], we observe an apparent threshold for dissipation around $v \simeq 0.6 c_{1 \mathrm{D}}^{(0)}$ close to the measured value $v \simeq 0.4 c_{1 \mathrm{D}}^{(0)}$.

\section{Conclusion}

In this article we have calculated the high-frequency spectrum of an elongated superfluid using WKB approximation. We showed that when subject to a periodic drive, dissipation occurs at all velocity, in contradiction with Landau's argument. In addition to vortex nucleation and transverse inhomogeneity of the cloud [19,20], this effect contributes significantly to the reduction of the apparent critical velocity observed in many experiments. Nevertheless, Landau-like features can be observed for an elongated superfluid excited by a narrow potentials oscillating at frequencies higher than the axial frequency, and lower than the transverse trapping frequency.

For low frequency excitations, the low-order harmonics couple to the discrete part of the spectrum, creating a weakly-damped coherent-oscillation of the low-lying modes of the superfluid, as for instance observed for in early BEC experiments [31] and dual Bose-Fermi superfluid counterflows [32]. On top of these oscillations, the higher-order harmonics will couple to the phonon-like sector, which dominates the sum appearing in Eq. (36) when the velocity is larger than the sound velocity, and will give rise to a Landau-like behaviour. Note that strictly speaking we can no longer treat the hydrodynamic modes of the superfluid as a continuous spectrum for $\Omega \simeq \omega_{z}$. However, at finite temperature, damping broadens the eigenmodes leading to a continuous-like spectrum when the decay rate becomes comparable to $\Delta \omega$. In the present work this broadening is introduced phenomenologically but a more quantitative understanding of thermal effects is therefore required to obtain a comprehensive understanding of the damping mechanism.

The authors thank I. Danaila, P. Parnaudeau, and the ultracold Fermi group for stimulating discussions. They acknowledge support from Région Ile de France (DIM IFRAF/NanoK), ANR (Grant SpiFBox) and European Union (ERC Grant ThermoDynaMix and CRITISUP2).

\section{References}

1. L. Landau. Theory of the superfluidity of helium ii. Phys. Rev., 60:356-358, Aug 1941.

2. Pavel A Cherenkov. Visible emission of clean liquids by action of $\gamma$ radiation. Doklady Akademii Nauk SSSR, 2:451, 1934.

3. W. Thomson. On ship waves. Institution of Mechanical Engineers, Proceedings, 38:409$434,1887$.

4. E. Raphaël and P.-G. de Gennes. Capillary gravity waves caused by a moving disturbance: Wave resistance. Phys. Rev. E, 53:3448-3455, Apr 1996.

5. J. Wilks and D.S. Betts. An introduction to liquid helium. Oxford science publications. Clarendon Press, 1987.

6. C Raman, M Köhl, R Onofrio, DS Durfee, CE Kuklewicz, Z Hadzibabic, and W Ketterle. Evidence for a critical velocity in a Bose-Einstein condensed gas. Physical Review Letters, 83(13):2502, 1999.

7. D. Miller, J. Chin, C. Stan, Y. Liu, W. Setiawan, C. Sanner, and W. Ketterle. Critical Velocity for Superfluid Flow across the BEC-BCS Crossover. Phys. Rev. Lett., 99(7):070402, August 2007.

8. Rémi Desbuquois, Lauriane Chomaz, Tarik Yefsah, Julian Léonard, Jérôme Beugnon, Christof Weitenberg, and Jean Dalibard. Superfluid behaviour of a two-dimensional Bose gas. Nature Physics, 8(9):645-648, 2012. 


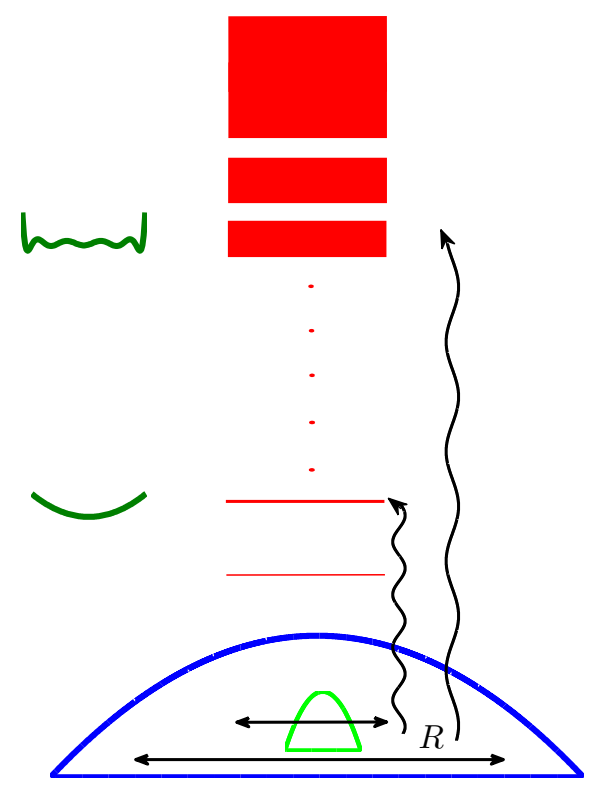

Fig. 4. Coupling to a low-frequency excitation. For $\Omega \simeq \omega_{z}$ the low-order harmonics of the excitation are coupled to the discrete part of the superfluid excitation spectrum. By contrast, the higher-order harmonics couple to the high frequency modes which are broadened by their finite lifetime and form a quasi-continuum.

9. K. C. Wright, R. B. Blakestad, C. J. Lobb, W. D. Phillips, and G. K. Campbell. Threshold for creating excitations in a stirred superfluid ring. Phys. Rev. A, 88:063633, Dec 2013.

10. Wolf Weimer, Kai Morgener, Vijay Pal Singh, Jonas Siegl, Klaus Hueck, Niclas Luick, Ludwig Mathey, and Henning Moritz. Critical velocity in the BEC-BCS crossover. Phys. Rev. Lett., 114:095301, Mar 2015.

11. Marion Delehaye, Sébastien Laurent, Igor Ferrier-Barbut, Shuwei Jin, Frédéric Chevy, and Christophe Salomon. Critical velocity and dissipation of an ultracold bose-fermi counterflow. Phys. Rev. Lett., 115:265303, Dec 2015.

12. Alberto Amo, Jérôme Lefrère, Simon Pigeon, Claire Adrados, Cristiano Ciuti, Iacopo Carusotto, Romuald Houdré, Elisabeth Giacobino, and Alberto Bramati. Superfluidity of polaritons in semiconductor microcavities. Nature Physics, 5(11):805-810, 2009.

13. R.P. Feynman. Chapter $\{\mathrm{II}\}$ application of quantum mechanics to liquid helium. volume 1 of Progress in Low Temperature Physics, pages 17 - 53. Elsevier, 1955.

14. Th Frisch, Yves Pomeau, and Sergio Rica. Transition to dissipation in a model of superflow. Physical review letters, 69(11):1644, 1992.

15. B. Jackson, J. F. McCann, and C. S. Adams. Dissipation and vortex creation in boseeinstein condensed gases. Phys. Rev. A, 61:051603, Apr 2000.

16. C. Nore, C. Huepe, and M. E. Brachet. Subcritical dissipation in three-dimensional superflows. Phys. Rev. Lett., 84:2191-2194, Mar 2000.

17. Woo Jin Kwon, Geol Moon, Sang Won Seo, and Y. Shin. Critical velocity for vortex shedding in a bose-einstein condensate. Phys. Rev. A, 91:053615, May 2015.

18. R. Combescot, M. Kagan, and S. Stringari. Collective mode of homogeneous superfluid Fermi gases in the BEC-BCS crossover. Phys. Rev. A, 74(4):042717, October 2006.

19. P. O. Fedichev and G. V. Shlyapnikov. Critical velocity in cylindrical bose-einstein condensates. Phys. Rev. A, 63:045601, Mar 2001. 
20. Pierre-Philippe Crépin, Xavier Leyronas, and Frédéric Chevy. Hydrodynamic spectrum of a superfluid in an elongated trap. EPL (Europhysics Letters), 114(6):60005, 2016.

21. Andrew G. Sykes, Matthew J. Davis, and David C. Roberts. Drag force on an impurity below the superfluid critical velocity in a quasi-one-dimensional bose-einstein condensate. Phys. Rev. Lett., 103:085302, Aug 2009.

22. Vijay Pal Singh, Wolf Weimer, Kai Morgener, Jonas Siegl, Klaus Hueck, Niclas Luick, Henning Moritz, and Ludwig Mathey. Probing superfluidity of bose-einstein condensates via laser stirring. Phys. Rev. A, 93(2):023634, 2016.

23. Guillaume Lang, Frank Hekking, and Anna Minguzzi. Dynamic structure factor and drag force in a one-dimensional strongly interacting bose gas at finite temperature. Phys. Rev. A, 91:063619, Jun 2015.

24. AD Chepelianskii, Frédéric Chevy, and Elie Raphael. Capillary-gravity waves generated by a slow moving object. Physical review letters, 100(7):074504, 2008.

25. John David Jackson. Classical electrodynamics. Wiley, 1999.

26. S Stringari. Dynamics of Bose-Einstein condensed gases in highly deformed traps. Phys. Rev. A, 58(3):2385, 1998.

27. E Zaremba. Sound propagation in a cylindrical Bose-condensed gas. Physical Review A, 57(1):518, 1998.

28. P. Capuzzi, P. Vignolo, F. Federici, and M. P. Tosi. Sound propagation in elongated superfluid fermionic clouds. Phys. Rev. A, 73:021603, Feb 2006.

29. W. Zwerger, editor. The BCS-BEC Crossover and the Unitary Fermi Gas, volume 836 of Lecture Notes in Physics. Springer, Berlin, 2012.

30. Milton Abramowitz and Irene A Stegun. Handbook of mathematical functions: with formulas, graphs, and mathematical tables, volume 55. Courier Corporation, 1964.

31. R Onofrio, C Raman, JM Vogels, JR Abo-Shaeer, AP Chikkatur, and W Ketterle. Observation of superfluid flow in a Bose-Einstein condensed gas. Physical Review Letters, 85(11):2228, 2000.

32. I Ferrier-Barbut, M. Delehaye, S. Laurent, A.T. Grier, M. Pierce, B.S Rem, F. Chevy, and C. Salomon. A mixture of Bose and Fermi superfluids. Science, 345:1035-1038, 2014 . 